\title{
Fire Monitoring System for Fire Detection Using ZigBee and GPRS System
}

\author{
D Kiran kumar ${ }^{1}$, G.Kishore ${ }^{2}$, T.V.Suresh kumar $^{3}$ \\ ${ }^{1}$ School of Electronics engineering, VIT University, Chennai campus \\ ${ }^{2,3}$ ECE dept Bharat Institute of Engg \& Tech
}

\begin{abstract}
Wireless Sensor Networks (WSN) is best suited for applications where continuous and long term data acquisition is required. Forest fire monitoring is one of such application where continuous monitoring of temperature and humidity is essential to detect the wildfire. Monitoring forest for wildfire detection is very mисh necessary to protect environment and to conserve forest wealth and habitats of biodiversity and livelihood of human. This paper presents an algorithm to detect the wildfire based on the changes occurring in humidity and temperature during fire and presents methodology based on ZigBee and GPRS wireless sensor network which provides low cost solution with long life time, low maintenance and good quality service as compared to the traditional method of wildfire detection. The hardware circuitry of proposed solution is based on Arduino board with ATmega328 microcontroller, temperature sensor and humidity sensor along with ZigBee and GPRS modules.
\end{abstract}

Keywords: WSN, ZigBee, GPRS, temperature and humidity sensors, Arduino board.

\section{Introduction}

Forest ecosystems are dynamic and complicated. Disturbance to any part of the network affects the balance of relationships and equally on the complete schemes either absolutely or negatively. When global climate change, drought and different conditions have caused occurrences of intense wildfire to extend, which may harm forest badly so once wildfire occurs it takes years to naturally recover. Sometimes wildfire becomes important for forests, however it additionally becomes a threat to them, thus sturdy policies and management are important to prevent wildfire. The best way to prevent such wildfire is to detect fire as early as possible [7]. To detect the fire main three environmental conditions one has to monitor are relative humidity, temperature and wind direction. Wind direction is useful in finding the direction in which fire can be spread. But relative humidity and temperature can detect the condition where fire can occur and exact situation where fire has occurred or not occurred [1].

Two factors which have direct influence on fire are relative humidity and temperature. Temperature is very essential factor in detecting the wildfire as it is a measure requirement in ignition and continuing the combustion process [6]. The old wood and hey takes radiated heat from sun to ignite them self. Sudden rise in temperature gives a hint in detection of wildfire [2] this sudden rise in temperature can also be happen at the time of sunrise. To avoid the confusion between the situations where sudden temperature rise occurs, we consider the next real time factor which is relative humidity. Humidity is nothing but moisture in the form of water vapour present in the atmosphere [6]. Relative humidity is the percentage ratio of actual water vapour contain to the amount of water vapour that would saturate the atmosphere at the same temperature [6]. If the humidity is low in environment, fuels normally transfers its moisture to the atmosphere and becomes flammable. Sudden decrease in humidity indicates that the fire alerts.

Normally fire closure occurs when relative humidity is less than $10 \%$, temperature is in between 89 $90 \mathrm{~F}$ and $\mathrm{RH}$ is 15 percent or less, temperature is in between $90-99 \mathrm{~F}$ and $\mathrm{RH}$ is 20 percent or less, temperature is higher than $100 \mathrm{~F}$ and $\mathrm{RH}$ is 25 percent or less [8].

Currently for wildfire detection different methods has been deployed such as patrols, satellite monitoring and observations from watch towers. As watch tower method is low cost and easy but it has many drawbacks such as need of trained labours, personal abound as carelessness of labours, absence from the post, limited coverage area. In other hand satellite monitoring is highly costly and comes with drawbacks as it is not effective as the images from satellite sometimes becomes useless due to pixel covered by clouds and also it needs long scanning cycle and resolution of saturated pixel dots of images is low[3]. WSN is solution in between cost and effectiveness. zigbee based sensor nodes in WSN monitors real time temperature and humidity data which effects in wildfire and sends the data to gateway nodes where the collected data is analysed and change in data is monitor. Depends on the change in data and proposed algorithm, the decision is taken by the gateway node which can send message to the forest department about detection of wildfire with the help of GPRS(General Packet Radio Service) system [1]. In this paper, the algorithm is given based on changes occurred in relative humidity and temperature to detect the fire. To monitor temperature and relative humidity 
sensors have been used. Temperature has been measured by LM35.

Temperature sensor and humidity is measured by HTM2500 and both values are verified with ATmega328 microcontroller. Arduino board with microcontroller ATmega328 has been chosen for this application due to its small packaging, low power consumption and low maintenance which is required in long term monitoring and difficult maintenance application as fire monitoring.

\section{A. Proposed Algorithm}

\section{Related Work}

The proposed solution is based on the algorithm which checks the changes occurred in relative humidity and temperature. In normal condition temperature and humidity values change shows cyclic behaviour [2] and temperature shows inverse proportion with relative humidity [1]-[3]. When fire is detected the above entities shows drastic change in the values. Current algorithm is based on state machines as shown in fig.

1. The state machine is made up of three basic state. The transition in state occurs when sudden rise in temperature or relative humidity occurs [2]. The zero state indicates the normal state, in this state the temperature is monitored continuously. This state is in self loop, when sudden rise in temperature occurs machine changes its state from state 0 normal states to the second state. To avoid ambiguity between rise in temperature due to fire and rise in temperature due to other reasons like sunrise or combustion of gases on sun's surface, the machine stays in state 1 . In state 1 , continuous monitoring of humidity and temperature is takes place. If the temperature is again rising continuously and humidity goes on decreasing then machine goes to state 3 which is indicated as fire detection state. If the temperature remains constant then machine goes back to the normal state.

In each state 0 , the average of the previous five values of temperature (Tavg) is calculated and which is checked against the new measured value which is $t$ (temperature), if the new measured value is exceeds 3 degree celsius in case of temperature then the change from state 0 to state 1 occurs. In state 1 , average of previous five values of humidity i.e. Havg is checked against current measured value $h$. If $h$ is falling then

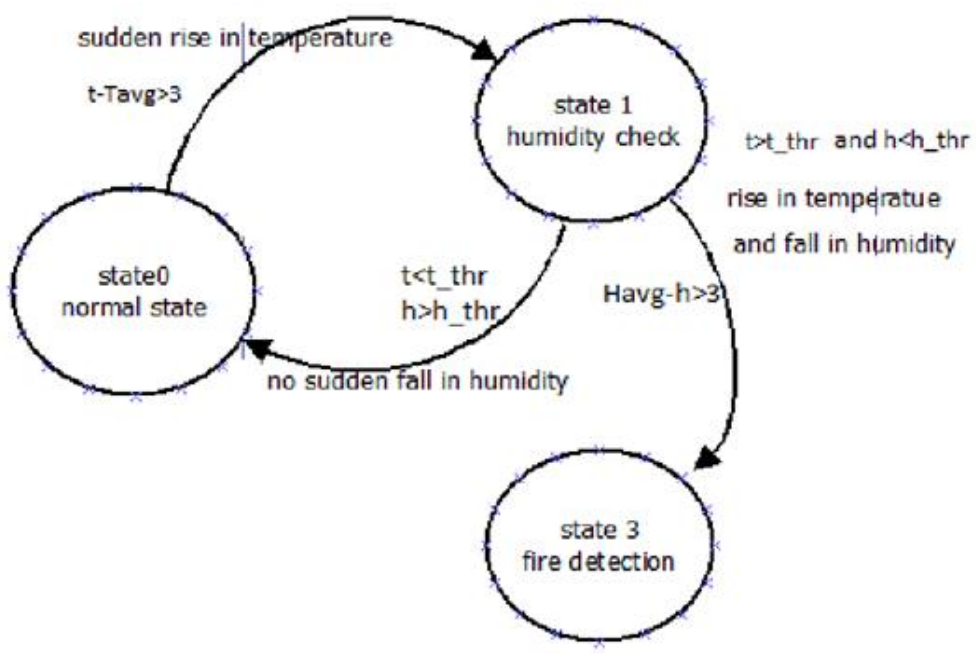

Fig.1 State diagram of proposed algorithm

state remains in state 1 and checks the predefined threshold values of temperature ( $\mathrm{t}$ _thr )and humidity (h_thr) if the temperature exceeds threshold and humidity goes below threshold then state change occurs from state 2 to state3

\section{B. Wireless sensornetwork}

Wireless sensor network is as shown in fig. 2. The sensor end point nodes are light in weight and can be mounted on trees, the end points are made with Arduino board interfaced with temperature sensor LM35 and HTM2500 the values are transferred to the gateway nodes via serial communication. For serial communication the ZigBee modules are used which are interfaced with ATmega328 in Arduino board. ZigBee transition is used for 3 meter range with low power of $3.3 \mathrm{v}$ supply.

Number of sensor nodes are arranged to cover the entire coverage, these ZigBee will work as routers in the zigbee network. The gateway node is also has Arduino interfaced with zigbee module. The gateway zigbee will act as coordinator. One coordinator with number of router is assigned the same PAN ID( personal area network ID). Number of such personal area networks can be implemented to cover the larger areas. In the gateway node, the microcontroller is loaded with algorithm which is designed for the project. , by using that 
algorithm, gateway node will take decision about wildfire detection. The same gateway node has GPRS functionality attached with it.

When fire is detected, the gateway node sends the message via GPRS to the forest department. Each gateway node has unique number of SIM card, from that the person in department can detect the location of gateway node. By providing the functionality of GPRS to the gateway node, one can monitor continuous data of humidity and temperature of particular location by activating data transfer via GPRS to internet service.

But to avoid power overload, method is using GPRS to send simple alert message to the forest department. GPRS has long range communication range but it is responsible for the most energy consumption in gateway node [1].
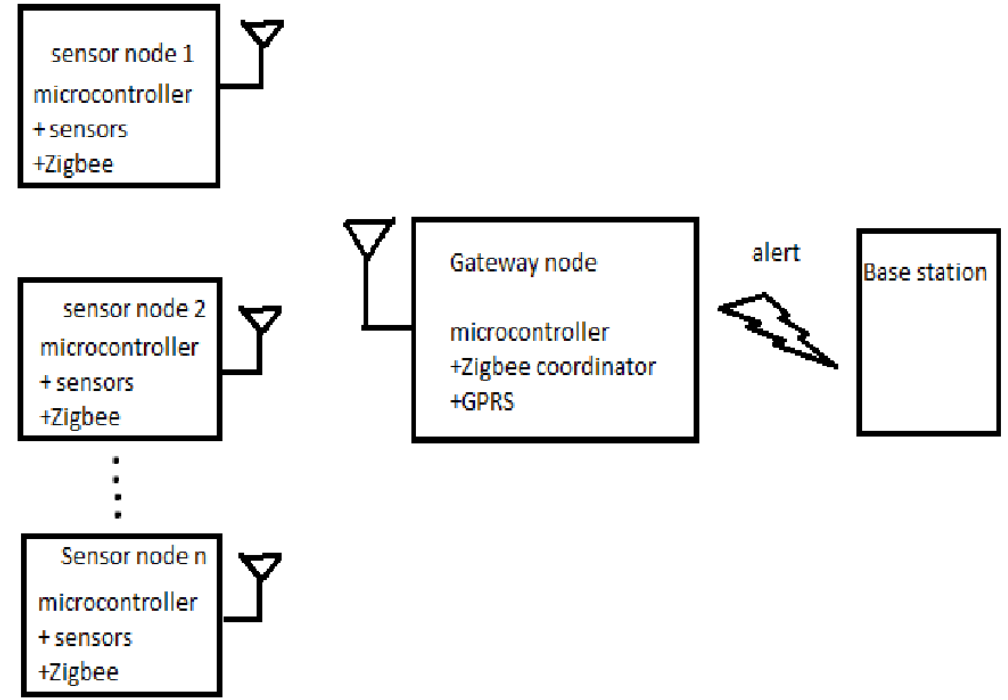

Fig.2. Block diagram of proposed work.

\section{Experimental Results}

The method have used the proposed algorithm and methodology explained as above for implementation of fire detection in forest. Before interfacing with the Arduino boards both the temperature and humidity sensors are calibrated for Arduino board with the help of reference sensors. The calibration factor for temperature and humidity sensors are defined in equation (1) and (2) respectively, where read_value indicates the respective reading obtained by sensors in Arduino UNO board.

Temperature $=(5.0 *$ read_value $* 100.0) / 1024$

The above measured value is gives temperature in degree Celsius.

Humidity $=(((($ read_value/1023)*5) $)-0.8) / 3.1) * 100$

The above humidity is measured in ppm.

The method have interfaced LM35 as temperature sensor and HTM2500 as humidity sensor and observed the behaviour of humidity and temperature at normal condition and at the time of fire. Fig. 3 shows the temperature and humidity behaviour graph obtained at different time and conditions.

Depends on the obtained observation at the time of fire we have decided the threshold of temperature and relative humidity. The experiment for the above project is verified with two sensor nodes and one gateway node. The interfacing of the board with the Arduino and zigbee is shown in fig. 4. The transmission of two routers to coordinator via zigbee has verified with the software XCTU. XCTU was used for the initial configuration of zigbee. This configuration includes setting of zigbee mode i.e. as router or coordinator, setting delay, destination address and PAN ID of network. The same software has used to check data transmission via terminal window. This coordinator data on XCTU terminal from two routers has shown in fig. 5.

At the gateway node one Arduino board has connected with Zigbee and another board has connected with GPRS, and both these boards are synchronised with simple pulse logic. That is when fire gets detected the first board simply triggers other board to turn on GPRS. 


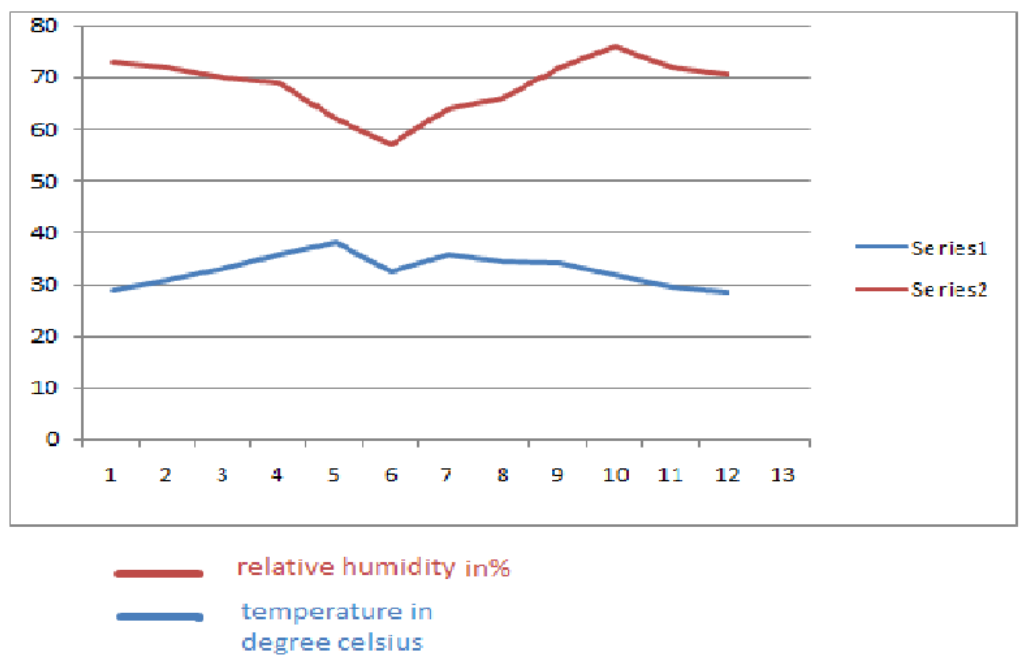

Fig. 3. Humidity and temperature behaviour during different time and condition of environment.

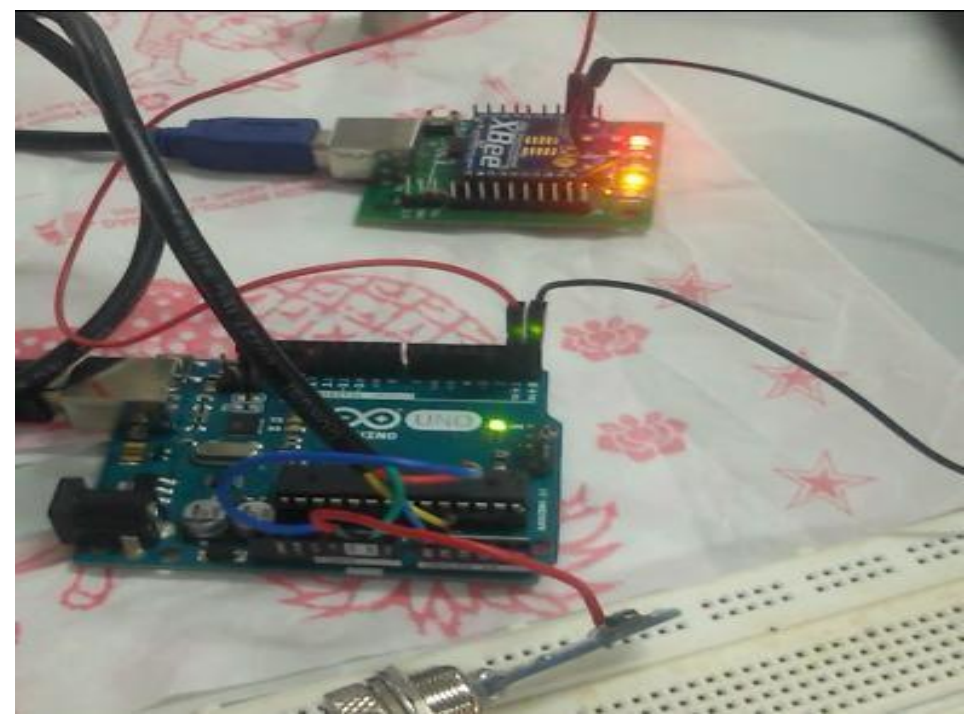

Fig. 4. Hardware interface of Arduino board and Zig Bee and sensors

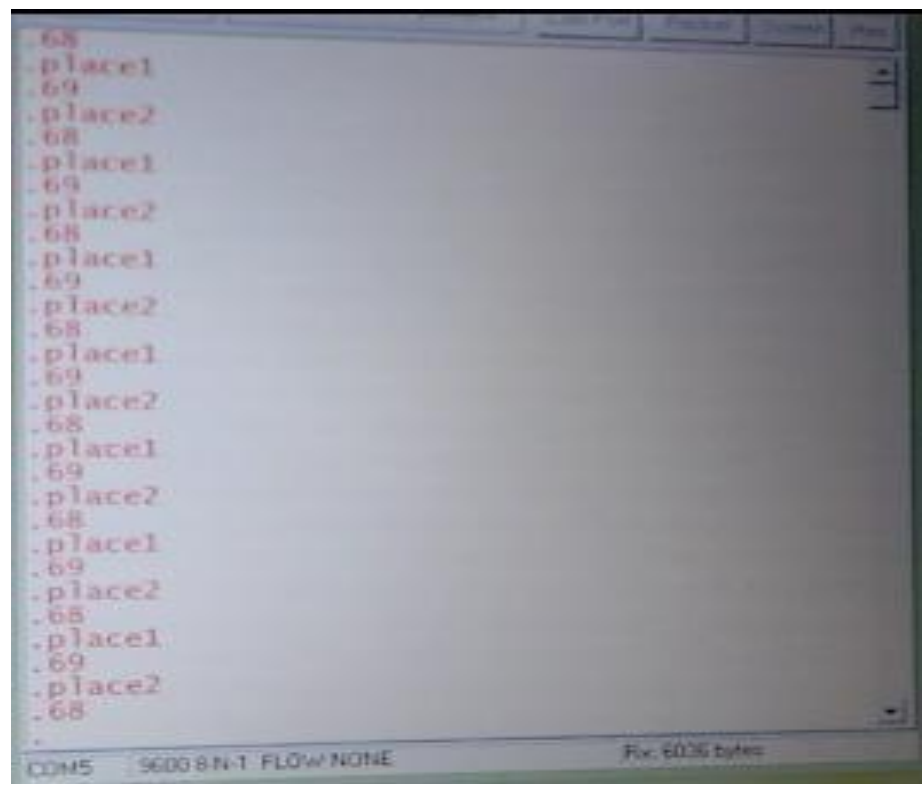

Fig. 5. XCTU terminal of gateway zigbee as coordinator from two different routers 


\section{Conclusions}

Forests are the backbone of ecosystems and conservation of these forests is our duty as part of this ecosystem. Forest systems are very dynamic and complex in nature, and hence to maintain the balance of the system is important. One of the threats to imbalance the system is wildfire. To detect and monitor this wildfire many methods are available like continuous watch from tower, satellite monitoring. But these methods come with different drawbacks. In this paper, the method has developed a methodology to overcome the drawbacks in previous methods. In this method, detection the fire by monitoring the parameters which affect the fire, they are temperature and humidity. Continuous monitoring of these two parameter helps us to detect the occurrence of wildfire. The method has included wireless sensor network to transfer the information. This network has included short range communication via zigbee and long range communication via GPRS. Short range communication has included for data exchange between different area of the forest and to process this data, where as long term communication has used to send the alert messages.

The unique SIM ID in GPRS helps to detect the exact location of the wildfire. This method is less complex and needs low maintenance, and useful in low power application.

\section{References}

[1]. Mihai T. Lazarescu, "Design of a WSN Platform for Long-Term Environmental Monitoring for IoT Apllication”,IEEE journal on engineering and selected topics in circuits and systems, volume 3, no 1, march 2013.

[2]. Arnoldo Díaz-Ramírez,LuisA.Tafoya, Jorge A. Atempa,PedroMejía- Alvarezb," Wireless Sensor Networks and Fusion Information Methods for Forest Fire Detection",Elsevier journals on The 2012 Iberoamerican Conference on Electronics Engineering and Computer Science

[3]. Junguo ZHANG, Wenbin LI, Ning HAN, Jiangming KAN, "Forest fire detection system based on a ZigBee wireless sensor network", published in Higher Education Press and Springer-Verlag 2008

[4]. K.Romer and F. Mattern, "The Design space of wireless sensor networks", IEEE wireless communication, vol.11,no.6,pp.5461,Dec2004

[5]. http://learnline.cdu.edu.au/units/env207/fundamentals/weather.html

[6]. http://www.auburn.edu/academic/forestry_wildlife/fire/weather_eleme nts.htm

[7]. https://www.americanforests.org/conservation-programs/forests-and- fire

[8]. www.larpd.org

[9]. Arduino : an open-source electronics prototyping platform , [online] Available: http://www.arduino.cc/

[10]. I. Dietrich and F. Dressler, "On the lifetime of wireless sensor networks", ACM Trans. Sensor Netw., vol.5, no. 1,pp. 5:1-5:39, feb 2009

[11]. A. Hasler, I. Talzi, C. Tschudin and S.gruber, "Wireless Sensor Networks in Permaforst research-concept, requirements, implementation and challenges", in Proc. $9^{\text {th }}$ Int. Conf. Permafrost, Jun 2008, Vol1, pp. 669-674

[12]. S.Verma, N. Chug and D.Gadre, "wireless sensor network for crop field monitoring" in recent trend Inf., Telecommun. Comput.,Mar2010, pp. 207-211 